\title{
Pre-Coded NRZ and Electrical Duo-Binary Transmission in C and O-band at Data Bit Rates up to $25 \mathrm{Gbit} / \mathrm{s}$
}

\author{
Justine Konopacki, Bertrand Le Guyader, Naveena Genay, Luiz Anet Neto, Philippe Chanclou, \\ and Didier Erasme* \\ Orange Labs, 2 avenue Pierre Marzin, 22307 Lannion, France \\ * LTCI, Télécom ParisTech, Université Paris-Saclay, 75013 Paris, France \\ Tel: (+33) 296072601 ,e-mail: justine.konopacki@orange.com
}

\begin{abstract}
In this paper we present real-time transmission performances up to $25 \mathrm{Gbit} / \mathrm{s}$ for optical access network. Our solution is based at transceiver side on pre-coded NRZ and electrical duo-binary modulations using limited electrical bandwidth DML in $\mathrm{C}$ and $\mathrm{O}$ band. At the receiver side, an electrical duo-binary receiver based on an $8 \mathrm{GHz}$ APD photodiode combined with an online duo-binary to binary converter is employed.

Keywords: pre-coded NRZ, electrical duo-binary, directly modulated laser, optical access network.
\end{abstract}

\section{DATA BIT RATES EVOLUTION IN THE OPTICAL ACCESS NETWORK}

Currently, optical access standards ([1-4]) are based on "on/off” keying Non Return to Zero (NRZ) modulation format with direct intensity modulation at the emitter and direct detection at the receiver side. The present evolution of the number of access network subscribers [5] and their behaviour in terms of daily network utilization is directly related to the continuous enhancement of the desired throughput per customer. This effect pushes up the demand for transmission rate [6]. In order to provide the network evolution, ITU-T defined NGPON2 standard [3], a technical solution based on Time and Wavelength Division Multiplexing (TWDM) which involves up to 8 channels working at $10 \mathrm{Gbit} / \mathrm{s}$ in downstream and/or upstream. Now, IEEE (NGE-PON) and FSAN/ITU-T (25G-PON) are working on technologies in order to define high data bit rate optical access solutions based on $25 \mathrm{Gbit} / \mathrm{s}$. In such context, systems based on an NRZ modulation format coupled to electrical equalization techniques and signal processing have been proposed [7,8]. More efficient modulation formats such as duo-binary modulation [9,10] and Pulse Amplitude Modulation (PAM-4) [10,11] are also considered. Whatever the field of application, optical access equipment have to be low-cost. Also it should take any possible opportunity to re-use optical components (Direct Modulated Lasers (DML) and Avalanche Photodiodes (APD)) previously involved in the $10 \mathrm{Gbit} / \mathrm{s}$-based access networks.

In this paper, we propose to consider a duo-binary reception Optical Line Terminal (OLT) for operation at rates up to $25 \mathrm{Gbit} / \mathrm{s}$ based on either a pre-coded NRZ (p-NRZ) or an Electrical Duo-Binary (EDB) incoming signal and an $8 \mathrm{GHz}$ APD photodiode. Section 2 describes EDB generation techniques. The transmission experimental setup is described in Section 3 and Section 4 compares the transmission results. Section 5 provides some conclusions and perspectives.

\section{EDB MODULATION FORMAT}

\subsection{Duo-binary signals generated via an electrical low-pass filter}

EDB modulation (Fig. 1a) is based on an NRZ sequence that is pre-coded thanks to a XOR gate. The pre-coded part is used first to easy recover the duo-binary data [12] and second to prevent error propagation [13]. Next the signal passes through a Finite Impulse Response (FIR) filter [14] which can be approximated as a $5^{\text {th }}$ order Bessel Low-Pass Filter (LPF) with a cut off frequency at half the bit rate.

The LPF attenuates abrupt transitions as "0 10 " and " 1001 " generating a third level in the pre-coded signal (Fig. 1b). The EDB signal could be generated at transceiver (Tx) side by introducing a duo-binary coder. It could be also generated at the receiver (Rx) side using a low electrical bandwidth receiver or both Tx and Rx side by using a low-bandwidth emitter and receiver. In our tests, we use an $8 \mathrm{GHz}$ APD with an electrical response represented Fig. 1a, which matches the $5^{\text {th }}$ order Bessel LPF response.

(a)

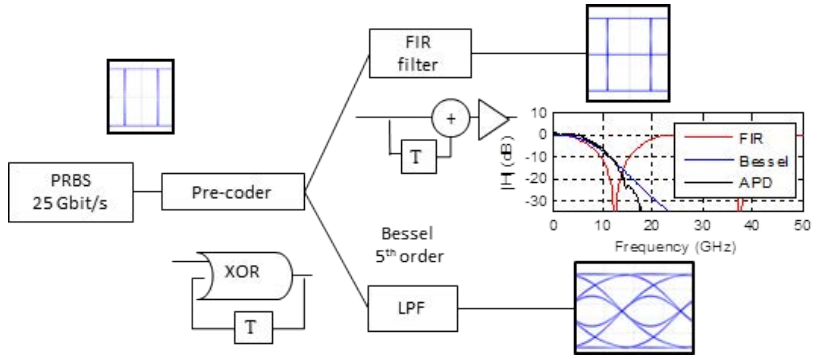

(b)

\begin{tabular}{|c|c|}
\hline PRBS & 101010110100100101 \\
\hline $\begin{array}{c}\text { XOR } \\
\text { out }\end{array}$ & 0110011011000111001 \\
\hline $\begin{array}{c}\text { FIR filter } \\
\text { out }\end{array}$ & $+10-10+100+10-1-10+1+10-10$ \\
\hline
\end{tabular}

Figure 1: (a) Simulated electrical duobinary signal generation at 25 Gbit/s;

(b) FIR filter and Low-Pass Filter (LPF) transfer function. 

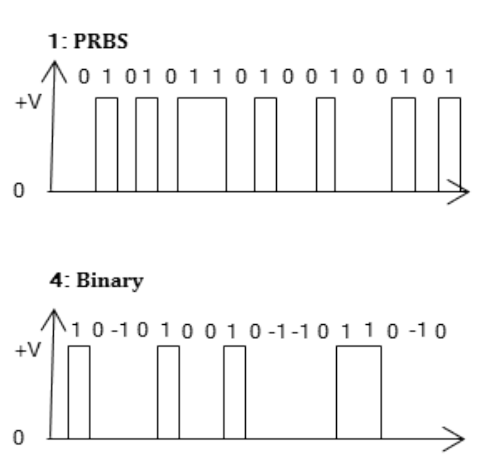

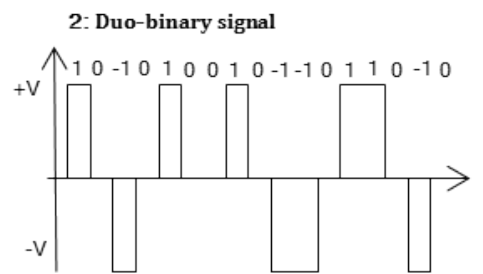

5: Binary

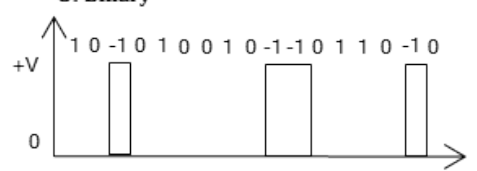

3: $\overline{\text { Duo-binary }}$ signal

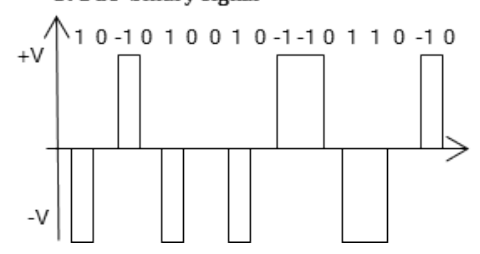

6: Binary

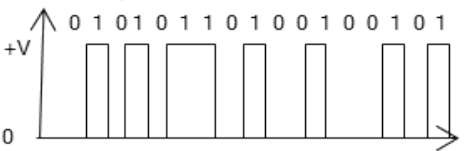

Figure 2. Duo-binary to NRZ decoder theoretical scheme.

\subsection{Duo-binary to NRZ decoder based on electrical components}

References [15-17] propose some offline processing techniques to decode an EDB signal. They are based on electrical comparators followed by a XOR gate.

In this paper, we propose an in line decoder which uses the two APD outputs ("D" and “ $\bar{D}$ "). Figure 2 represents the logic corresponding to our simple in line duo-binary to NRZ decoder presented Fig. 3a. Our scheme consists in using two complementary output ports of an APD providing the EDB signal " $D$ " as well as the complementary signal " $\bar{D}$ ". Two limiting amplifiers are used to select out the positive part of the EDB signal (Figs. 2-4 and 2-5 and points 4 and 5 in Fig. 3a). The top (resp. bottom) limiting amplifier select out symbol " $+1 "$ (resp. "-1") from all other symbols (i.e. "0" and "-1, resp. " 0 " and " $+1 ")$. Finally (point 6 in Fig. 3a), a XOR gate builds back the initial NRZ signal. A delay line need be added in the scheme in order to adjust the delay between the two arms of our in line decoder.

\section{EDB AND p-NRZ TRANSMISSION EXPERIMENTAL SETUP FOR DATA BIT RATES UP TO 25 GBIT/S}

The experimental setup used for electrical generating EDB and p-NRZ signals for optical transmitting tem and for reception scheme is depicted in Fig. 3a.

In the case of the emitter "Tx1", a $2^{31}-1$ PRBS NRZ electrical signal generated by a Pulse Pattern Generator (PPG) is converted into an EDB sequence thanks to a duo-binary encoder working up to 28 Gbit/s. The EDB signal is next amplified up to $2.7 \mathrm{Vpp}$ via a $35 \mathrm{GHz}$ RF-amplifier and is used to modulate DMLs characterized by an emission wavelength either located in C-band or O-band.

For the second emitter "Tx2", a $2^{15}-1$ pre-coded PRBS NRZ signal is directly applied to the same DMLs as used in the case of emitter "Tx1".

a)

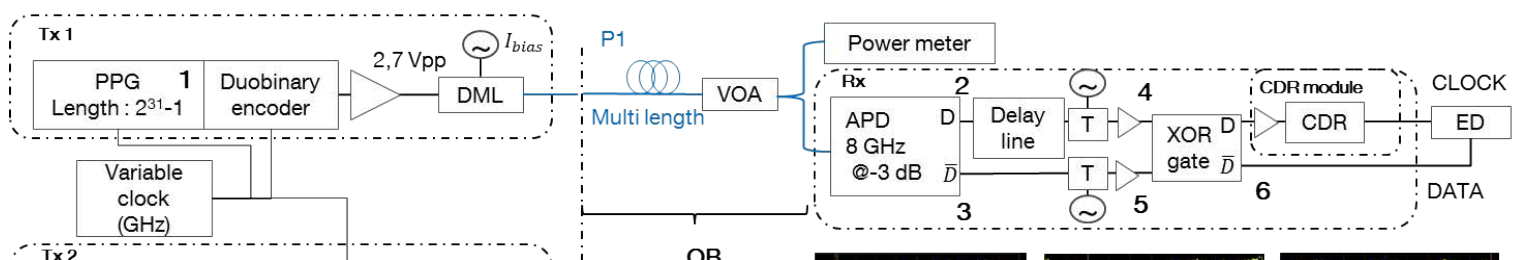

OB

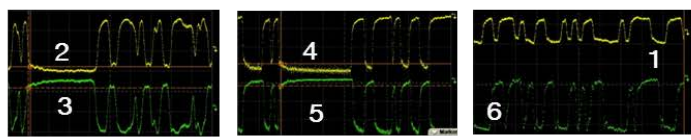

b)

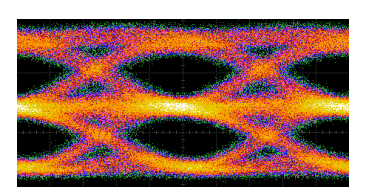

1. EDB at Tx and EDB Rx at $25 \mathrm{Gbit} / \mathrm{s}\left(\mathrm{P}_{\text {inAPD }}=-10 \mathrm{dBm}\right)$

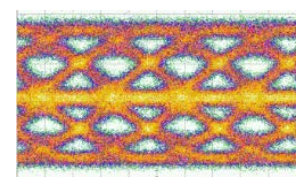

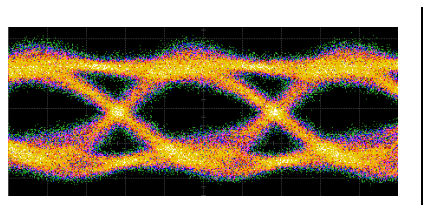

2. p- NRZ Tx and EDB Rx at $25 \mathrm{Gbit} / \mathrm{s}\left(\mathrm{P}_{\mathrm{inAPD}}=-10 \mathrm{dBm}\right)$

Figure 3: a) EDB and p-NRZ emitters with $E D B$ detection and in line decoder;

b) Eye diagrams in O-band at Tx and Rx side in back-to-back (BtB).

The C-band DML has $14 \mathrm{GHz}$ electrical bandwidth and emits $11.5 \mathrm{dBm}$ for a $100 \mathrm{~mA}$ bias current $\left(I_{\text {bias }}\right)$. The O-band DML is characterized by a $16 \mathrm{GHz}$ electrical bandwidth and an output optical power of $10.5 \mathrm{dBm}$ for $I_{\text {bias }}=77 \mathrm{~mA}$. Various optical fiber lengths (up to $40 \mathrm{~km}$ ) are used for the optical transmission and a Variable 
Optical Attenuator (VOA) allows tuning the optical power at the input of the receiver varying the Optical Budget (OB). For all the output optical power used, non-linear effects such as Stimulated Brillouin Scattering remain negligible. Rayleigh backscattering is also measured.

The receiver consists in an $8 \mathrm{GHz}$ electrical bandwidth APD equipped with an integrated trans-impedance amplifier. Figure $3 \mathrm{~b}$ includes some eye diagram assessment of the O-band transmissions. In the case of a transmitted EDB signal, the APD electrical bandwidth has negligible influence on the detected signal (Fig. 3b1). On the other hand for a $25 \mathrm{Gbit} / \mathrm{s}$ p-NRZ optical signal, the APD electrical bandwidth filters the signal and transforms it into duo-binary (Fig. 3b2). The APD outputs "D" output is connected to a first $28 \mathrm{GHz}$ limiting amplifier via a $26 \mathrm{GHz}$ delay line and " $\bar{D}$ " output directly to a second $28 \mathrm{GHz}$ limiting amplifier. At the photodiode output, a signal offset always allows to select the EDB positive part. Outputs of the amplifiers are connected to a $28 \mathrm{GHz}$ XOR gate and delivers out a NRZ signal (Fig. 3a point 6). A $32 \mathrm{GHz}$ Clock and Data Recovery (CDR) unit extracts the clock used to synchronize the Error Detector (ED).

\section{TRANSMISSION PERFORMANCES IN O AND C-BAND}

Figures $4 \mathrm{a}$ and $4 \mathrm{~b}$ summarize C-band and O-band transmission performances in the case of an EDB ("Tx1") and a p-NRZ ("Tx2") modulation format respectively. Performances are evaluated for data bit rates up to 25 Gbit/s. For each transmitter, Back-to-Back (BtB) performances are given at $10 \mathrm{Gbit} / \mathrm{s}$ for a $2^{31}-1$ PRBS NRZ signal. Following penalties are given with respect to these for a BER of $10^{-4}$.

Figure 4a displays BtB performances mainly as transmission is rapidly limited by chromatic dispersion in C-band. A $20 \mathrm{Gbit} / \mathrm{s}$ p-NRZ transmission is performed for $10 \mathrm{~km}$ of optical fiber: a $28 \mathrm{~dB}$ optical budget can be achieved for a BER of $10^{-4}$. C-band p-NRZ BtB penalty is $2 \mathrm{~dB}$ when the data bit rate increases from 20 Gbit/s to $25 \mathrm{Gbit} / \mathrm{s}$. This is due to the $8 \mathrm{GHz}$ electrical bandwidth limitation of the APD. In C-band, EDB and p-NRZ modulation format present equivalent BtB performances at $20 \mathrm{Gbit} / \mathrm{s}$.

In O-band, it is possible to achieve $40 \mathrm{~km}$ of propagation at $20 \mathrm{Gbit} / \mathrm{s}$ or $25 \mathrm{Gbit} / \mathrm{s}$ as chromatic dispersion effect is very limited. Measurements are done with both an EDB and a p-NRZ modulation format. In the case of the p-NRZ modulation format, the penalty is lower than $1 \mathrm{~dB}$ between the $\mathrm{BtB}$ and the $40 \mathrm{~km}$ performances for both $20 \mathrm{Gbit} / \mathrm{s}$ and $25 \mathrm{Gbit} / \mathrm{s}$. The $\mathrm{OB}$ is about $28 \mathrm{~dB}$ at $20 \mathrm{Gbit} / \mathrm{s}$ and $26.5 \mathrm{~dB}$ at $25 \mathrm{Gbit} / \mathrm{s}$ (for a BER of $10^{-4}$ ). EDB transmission achieves an $\mathrm{OB}$ of $30 \mathrm{~dB}\left(\mathrm{BER}=10^{-4}\right)$ at $20 \mathrm{Gbit} / \mathrm{s}$ with $40 \mathrm{~km}$ of optical fiber, BtB performances are the same but not represented here. EDB performances become worse in the case of a $25 \mathrm{Gbit} / \mathrm{s}$ data bit rate, $40 \mathrm{~km}$ propagation but still allow for an OB of $23.5 \mathrm{~dB}$ for a BER of $10^{-3}$.

At $25 \mathrm{Gbit} / \mathrm{s}$, EDB transmissions are limited by the electrical bandwidth of the APD receiver that filters the signal and deteriorates the decoded transmitted signal. In C-band (resp. O-band), it generates a BER error floor of $10^{-3}$ (resp. $5 \times 10^{-4}$ ).

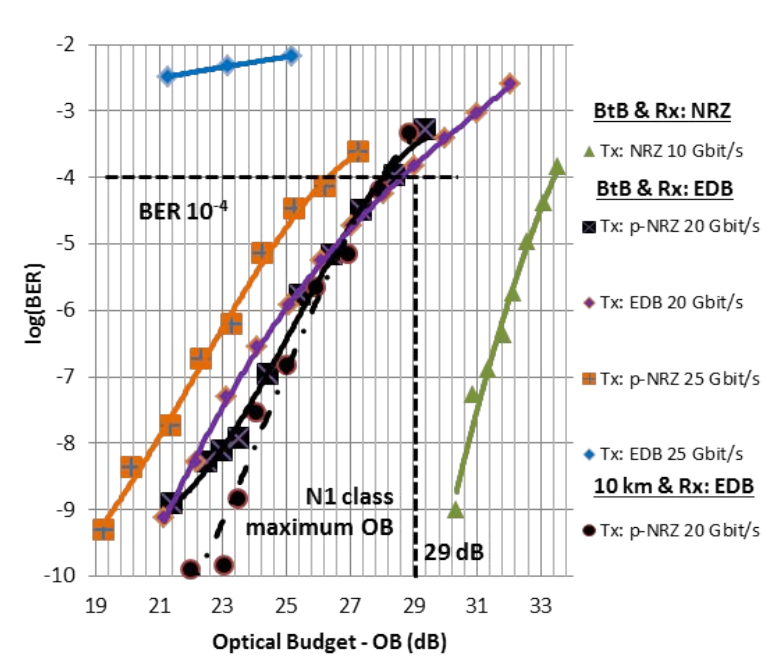

a) C-band transmissions performances

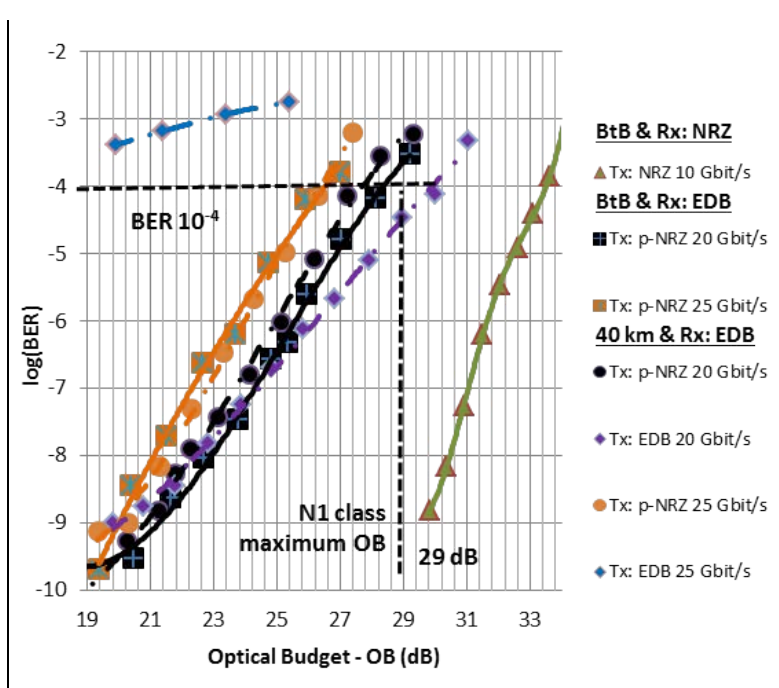

b) O-band transmissions performances

Figure 4. $C$ and $O$-band transmissions performances comparison according $E D B / p-N R Z$ and optoelectronic $E D B$ receiver.

\section{CONCLUSIONS AND PERSPECTIVES}

We demonstrated an O-band transmission up to $40 \mathrm{~km}$ with a $26.5 \mathrm{~dB}$ optical budget at $25 \mathrm{Gbit} /$ based on a pNRZ emission using a $14 \mathrm{GHz}$ low-bandwidth electrical DML. The receiver is using a classical $8 \mathrm{GHz}$ APD photodiode and a real-time EDB to NRZ converter based on limiting amplifiers and a XOR gate. This solution 
could be quite convenient for a low cost upstream architecture of a 25G-PON solution. In O-band we also demonstrate that $28 \mathrm{~dB}$ optical budget can be achieved for a $20 \mathrm{Gbit} / \mathrm{s}$ data bit rate with either a p-NRZ or EDB transmission. In C-band, transmission performances are much worse due to chromatic dispersion effects. However, p-NRZ transmission at $20 \mathrm{Gbit} / \mathrm{s}$ is demonstrated for a fiber length up to $10 \mathrm{~km}$ and a $28 \mathrm{~dB}$ optical budget. Performances in $\mathrm{C}$-band could still be improved by pre-equalization and electrical mitigation techniques.

\section{REFERENCES}

[1] ITU-T.G.984, "Gigabit-capable passive optical networks (GPON): General characteristics”, ITU-T, Mar. 2008.

[2] ITU-T.G.987, “10-Gigabit-capable passive optical networks (XG-PON): General requirements”, ITU-T, Jan. 2010

[3] ITU-T.G.989, “40-Gigabit-capable passive optical networks (NG-PON2): General requirements”, ITU-U, Mar. 2013.

[4] IEEE P802.3av, "Physical Layer Specifications and Management Parameters for $10 \mathrm{~Gb} / \mathrm{s}$ Passive Optical Networks", IEEE, Jun. 2009.

[5] Cisco, "Cisco Visual Networking Index: Forecast and Methodology, 2011-2016", 2012.

[6] Fiber to the Home Council Europe, "FTTH subscribers in Europe: nearly 15 million homes!" in Press release, 2015.

[7] Jin Wang and J. M. Kahn, "Performance of electrical equalizers in optically amplified OOK and DPSK systems," IEEE Photonics Technology Letters, vol. 16, no. 5, 2004.

[8] P. Venugopal, Y.S.V.S.R. Karthik, and J. Rudra,"10Gbps optical line using electronic equalizer and its cost effectiveness," International Journal of Engineering and Technology (IJET), vol. 5, no. 4, 2013.

[9] A. Lender, "The duobinary technique for high-speed data transmission," 1963.

[10] C. Sun, S. H. Bae, and H. Kim, "Transmission of 28-Gb/s duobinary and PAM-4 signal using DML for optical access network," IEEE Photonics Technology Letters, 2016.

[11] X. Li, S. Zhou, H. Ji, M. Luo, Q. Yang, L. Yi, R. HU, C. Li, S. Fu, A. Alphones, W.D. Zhong, and C. Yu, "Transmission of 4x28-Gb/s PAM-4 over 160-km single mode fiber using 10G-class DML and photodiode," in Proc. OFC, 2016, paper W1A.5.

[12] T. Ono, Y. Yano, K. Fukuchi, T. Ito, H. Yamazaki, M. Yamaguchi, and K. Emura, "Characteristics of optical duobinary signals in terabit/s capacity, high-spectral efficiency WDM systems ," Journal of Lightwave Technology, vol. 16, no. 6, 1998.

[13] R.D. Gitlin, J. Hayes, and S.B. Weinstein, “Data Communications Principles,” Applications of Communications Theory, Series Editor: R. W. Lucky, Springer Science \& Business Media, 2012.

[14] K. Rupinder and D. Sanjeev, "Duobinary modulation format for optical system - A review," International Journal of Advances Research in Electrical, Electronics and Instrumentation Engineering, vol. 3, no. 8, 2014.

[15] V. Houtsma, D. van Veen, A. Gnauck, and P. Iannone, “APD-based duo-binary direct detection receivers for 40 Gbps TDM-PON," in Proc. OFC, 2015, paper Th4H.1.

[16] Z. Li, L. Yi, X. Wang, and W. Hu, "28 Gb/s duo-binary signal transmission over $40 \mathrm{~km}$ based on $10 \mathrm{GHz}$ DML and PIN for $100 \mathrm{~Gb} / \mathrm{s}$ PON," Optics Express, vol. 23, no. 16, 2015.

[17] D. van Veen, V. Houtsma, P. Winzer, and P. Vetter, "26-Gbps PON transmission over 40-km using duobinary detection with a low cost 7-GHz APD-based receiver," in Proc. ECOC, 2012. 